\title{
Dependence of energy barrier reduction on collective excitations in square artificial spin ice: A comprehensive comparison of simulation techniques
}

\author{
Sabri Koraltan $\odot,{ }^{1, *}$ Matteo Pancaldi $\odot,{ }^{2}$ Naëmi Leo $\odot,{ }^{3}$ Claas Abert $\odot,{ }^{1}$ Christoph Vogler, ${ }^{1}$ Kevin Hofhuis $\odot, 4,5$ \\ Florian Slanovc, ${ }^{1}$ Florian Bruckner, ${ }^{1}$ Paul Heistracher $\odot,{ }^{1}$ Matteo Menniti $\odot,{ }^{3}$ Paolo Vavassori $\odot,{ }^{3,6}$ and Dieter Suess ${ }^{1}$ \\ ${ }^{1}$ Christian Doppler Laboratory, “Advanced Magnetic Sensing and Materials”, University of Vienna, Waehringer Strasse 17, \\ 1090 Vienna, Austria \\ ${ }^{2}$ Department of Physics, Stockholm University, 10691 Stockholm, Sweden \\ ${ }^{3}$ CIC nanoGUNE BRTA, 20018 Donostia-San Sebastián, Spain \\ ${ }^{4}$ Laboratory for Mesoscopic Systems, Department of Materials, ETH Zurich, 8093 Zurich, Switzerland \\ ${ }^{5}$ Laboratory for Multiscale Materials Experiments, Paul Scherrer Institute, 5232 Villigen PSI, Switzerland \\ ${ }^{6}$ IKERBASQUE, Basque Foundation for Science, 48013 Bilbao, Spain
}

(Received 6 June 2020; revised 30 July 2020; accepted 30 July 2020; published 12 August 2020)

\begin{abstract}
We perform micromagnetic simulations to study the switching barriers in square artificial spin ice systems consisting of elongated single domain magnetic islands arranged on a square lattice. By considering a double vertex composed of one central island and six nearest neighbor islands, we calculate the energy barriers between two types of double vertices by applying the simplified and improved string method. We investigate by means of micromagnetic simulations the consequences of the neighboring islands, the inhomogeneities in the magnetization of the islands and the reversal mechanisms on the energy barrier by comparing three different approaches with increasing complexity. The micromagnetic models, where the string method is applied, are compared to a method commonly in use, the mean barrier approximation. Our investigations indicate that a proper micromagnetic modeling of the switching process leads to significantly lower energy barriers, by up to $35 \%$ compared to the mean-barrier approximation, so decreasing the expected average life time up to seven orders of magnitude. Hereby, we investigate the influence of parallel switching channels and the conceptional approach of using a mean-barrier to calculate the corresponding rates.
\end{abstract}

DOI: 10.1103/PhysRevB.102.064410

\section{INTRODUCTION}

Artificial spin ice (ASI) systems are lithographically patterned lattices of elongated single domain magnetic islands [1-4]. In two-dimensional ASI systems, the magnetic islands are arranged in vertices and build a frustrated lattice due to the competing magnetostatic interactions among the islands $[1,5-$ 8]. A possible ASI system, where four islands build a vertex and are arranged on a square lattice, is called square artificial spin ice (sASI) [1,2]. Such a lattice is illustrated in Fig. 1(a). In the absence of external magnetic fields, the islands are magnetized along their long axis due to shape anisotropy, which limits the possible configurations in a vertex to $2^{4}=16$ macroscopic configurations.

Figure 1(b) shows the four types of vertex configurations in a sASI, where the energy of each vertex increases with its assigned number.

The ground state is represented by Type I vertices, since in a sASI lattice with alternating Type I vertices the stray fields between four magnetic islands arranged in a square build closed loops [9], minimizing the total energy of the system. Even though both Type I and Type II vertices obey the ice rule [2], where two magnetizations are pointing to the center

\footnotetext{
*sabri.koraltan@univie.ac.at
}

and two away from it, the latter have an increased energy due to unequal distance of the four elements forming a vertex. Type III vertices show even higher energies, where three magnetizations are pointing towards the center and one away or vice versa. This vertex configuration displays a net magnetic charge $Q_{m}= \pm 2 q_{m}$, where $q_{m}$ is the magnetic charge residing at the end of each magnetized island $[2,4]$. Type IV vertices contain magnetic islands with the magnetization pointing all to the center or away from it. This type has the highest energy level, and thus, is the most excited configuration, showing a net magnetic charge $Q_{m}= \pm 4 q_{m}$.

Both experiments [10] and simulations [11,12] have been performed to analyze the thermal annealing and domain dynamics in these structures. Furthermore, it has been experimentally observed that the ground state can be achieved after thermal relaxation, where the lattice has been subject to temperatures $T$ satisfying $T_{B}<T<T_{\text {Curie }}$, where $T_{B}$ denotes the blocking temperature above which the magnetizations of the islands are fluctuating on the time scale of the measurement [7,13-15], and $T_{\text {Curie }}$ denotes the Curie temperature. In this case, the islands start to switch their overall magnetic orientation along the long axis due to thermal activation, enabling the system to evolve towards the energetically favored ground state with Type I vertices. The temporal evolution of the thermally activated relaxation can be described by standard (Bortz-Kalos-Lebowitz algorithm [16]) kinetic Monte Carlo 


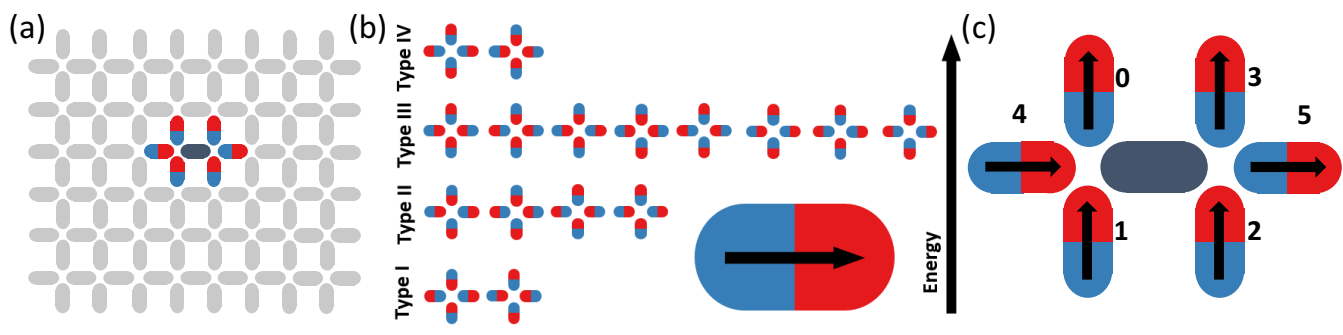

FIG. 1. Square artificial spin ice lattice. (a) Schematic illustration of a sASI lattice with a highlighted in color double vertex. (b) Vertex configurations in a sASI. Possible arrangements of the magnetizations of the islands in a square vertex, where the black arrow of the large island indicates the direction of the magnetization. All configurations that are assigned to the same vertex type have equal energies. (c) Schematic illustration of the double vertex from (a) with the central island (dark), being the island of interest in the energy barrier calculations and the neighboring islands enumerated with the island identification number $j$ to calculate the configuration number, as given later by Eq. (2).

simulations $[15,17,18]$ that require the energy barriers of the various switching processes as an input. Thus the ability to accurately determine the energy barriers to be overcome in order to switch the magnetization of the magnetic islands are of key importance.

By using the mean-barrier approximation to calculate the switching barriers, the experimentally observed relaxation mechanisms, and thus the dynamics and the ordering of the system, can only be artificially reproduced [15,18-21]. Namely, in order to reproduce the relaxation timescale observed experimentally, the energy barriers need to be artificially reduced. Such a reduction is usually ascribed to extrinsic factors like fabrication defects, reduction of the Curie temperature $T_{\text {Curie }}$ [22] or saturation magnetization $M_{s}$ [7].

In this work, we study the dependence of the energy barriers on collective excitations in a square lattice by performing micromagnetic simulations.

We apply the simplified and improved string method (SISM) [23] to investigate if the commonly reported barrier reduction is ascribable, and to what extent, to intrinsic fundamental physics related to the thermally induced reversal rather than a mere effect of extrinsic factors by comparing three different models.

We find that these reductions can be ascribed to the preferential switching direction of the magnetizations in a magnetostatic environment, as well as nonuniform contributions to the moment reversal.

This paper is organized as follows. The simplified and improved string method is presented and the application on a double vertex is described in Sec. II. The considered geometries and sASI material parameters are introduced in Sec. III. In the Secs. III A-III C we describe the three investigated models. We discuss our findings and the results of our simulations in Sec. IV, where we analyze in detail the differences in the reversal mechanisms, rotation directions, energy barriers, corresponding switching rates and different material parameters. We summarize our conclusions in Sec. V.

\section{SIMPLIFIED AND IMPROVED STRING METHOD}

In order to analyze the dependence of collective excitations on the energy barriers, we use the SISM [23] to calculate the energy barrier between two magnetic states.

This method consists of three steps. In the first step, we create an initial path for the switching event. For this purpose, we choose a coherent rotation with the uniform magnetization $\boldsymbol{m}_{\text {ini }}$ as our initial state. The final state of the initial path is represented by $\boldsymbol{m}_{\mathrm{fin}}$. This path is discretized by 21 distinct magnetization configurations, which sample the continuous transition path in an equidistant fashion with respect to an appropriate norm [23]. In a second step, each of these 21 magnetization configurations is evolved a certain amount towards its nearest energetic minimum, and in a final step, the 21 magnetization states are rearranged along the path in order to restore the equidistant discretization of the path. The last two steps are repeated until we obtain the minimum energy path (MEP). This final path represents the lowest energy path and equally, the most probable and favorable way to switch between initial and final magnetization states with respect to the given initial path. Figure 2 is an exemplary illustration for the evolution of the energy paths to obtain the MEP. In agreement with transition-state theory [24,25] the energy barrier to switch the magnetization from $\boldsymbol{m}^{\text {ini }}$ to $\boldsymbol{m}^{\text {fin }}$ is obtained by

$$
\Delta E=E^{\text {saddle }}-E^{\text {ini }},
$$

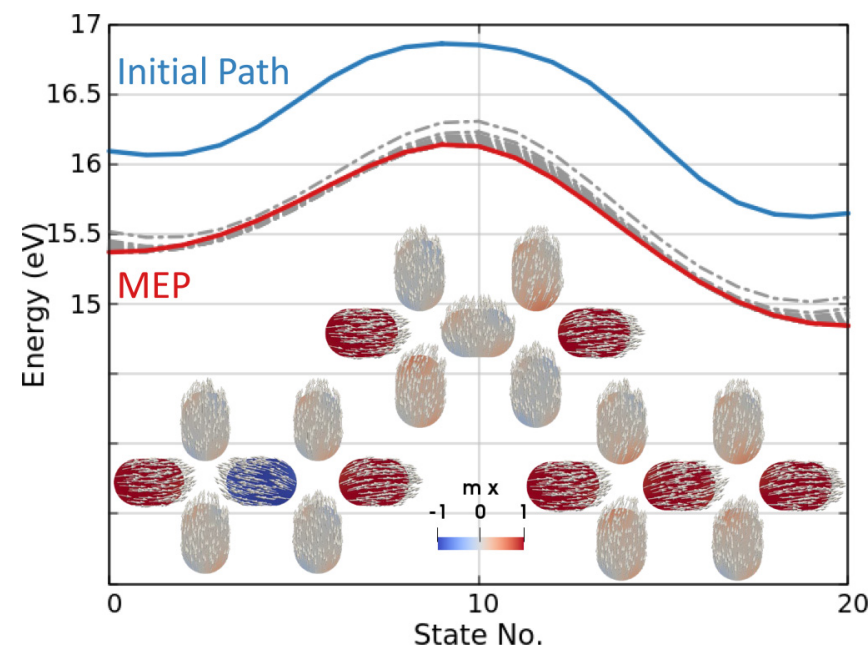

FIG. 2. Evolution of the energy paths during the SISM. For the configuration $c=63$ illustrated in Fig. 1(c), the initial path (blue), represented by a coherent rotation, evolves towards the MEP (red) via the intermediate energy paths (gray). Inset figures represent the magnetization states for State No. $=0$ (left), State No. $=10$ (center) and State No. $=20$ (right) of the MEP. 
TABLE I. Summary of the considered models. The environment describes the magnetization of the NN islands and the central moment the magnetization of the central island during the switching event.

\begin{tabular}{llll}
\hline \hline MODEL & MODEL 1 & MODEL 2 & MODEL 3 \\
\hline Description & Mean-barrier & Uniform environment & Full micromagnetic model \\
Environment & Uniform & Uniform & Dynamically relaxed \\
Central island & Uniform & Dynamically relaxed & Dynamically relaxed \\
Reversal mechanism & Coherent rotation & Minimum energy path & Minimum energy path \\
\hline \hline
\end{tabular}

where $E^{\text {saddle }}$ is the energy corresponding to the saddle point of the MEP and $E^{\text {ini }}$ is the energy of the initial state of the MEP.

The considered energy contributions for the micromagnetic modeling are described in Appendix A.

\section{MODELING}

In this paper, we study the energy barriers between different vertex types of sASI. In particular, we show the influence of the nearest neighbor $(\mathrm{NN})$ islands on the energy barrier by computing the MEPs with different approaches. The considered models are summarized in Table I. To obtain a direct comparison between the models, we use magnum.fe [26], a finite element method based micromagnetic simulation code, to calculate the minimum energy path by applying the SISM. We generate all finite element meshes using Gmsh [27].

We consider magnetic islands similar to those used to perform kinetic Monte Carlo simulations in literature, for which the energy barriers needed to be reduced with respect to those arising using the geometrical and physical properties of the nanoelements to reproduce the experimentally observed relaxation. Our islands have a length $L=150 \mathrm{~nm}$, a width $W=100 \mathrm{~nm}$, an edge-to-edge gap $g=90 \mathrm{~nm}$, and a thickness $t=3 \mathrm{~nm}$.

Furthermore, we use material parameters similar to bulk permalloy at $T=300 \mathrm{~K}$, with saturation magnetization $M_{\mathrm{s}}=$ $790 \mathrm{kA} / \mathrm{m}$, exchange stiffness constant $A=13 \mathrm{pJ} / \mathrm{m}$ and vanishing uniaxial anisotropy constant $K=0$.

In the following, we study the energy barrier for the switching event of the magnetization of the central island in a double vertex. Figure 1(c) illustrates such a double vertex with the central island as the island of interest.

In order to effectively label the 64 possible magnetization configurations of the $\mathrm{NN}$ islands, we introduce the integer parameter $c$ defined by

$$
c=\sum_{j=0}^{5} b_{j} 2^{j} .
$$

The factor $b_{j}$ is obtained by

$$
b_{j}=\left\{\begin{array}{ll}
0, & \text { if } m_{k}=-1 \\
1, & \text { if } m_{k}=+1
\end{array},\right.
$$

where $m_{k}$ is positive, if the magnetic moment points to the right (up) for horizontal (vertical) islands, as shown in Fig. 1(c). The index $j$ denotes the $\mathrm{NN}$ island identification number as illustrated in Fig. 1(c). The arrows in Fig. 1(c) show the magnetization of the neighboring islands in the configuration $c=63$.

\section{A. Model 1. Mean-barrier approximation}

For the first model, we consider the mean-barrier approximation when the NN islands are present and uniformly magnetized. Motivated by the symmetry properties of the strayfield interaction and Zeeman energies, the mean barrier $[15,18-21]$ of the switching is described by

$$
\Delta E^{\text {mean }}=\Delta E^{\text {isol }}+\frac{1}{2}\left(E^{\mathrm{fin}}-E^{\mathrm{ini}}\right),
$$

where $\Delta E^{\text {isol }}$ is the energy barrier to switch the magnetization of one isolated nanostructure, $E^{\text {fin }}$ the energy of the final state corresponding to the switched double vertex and $E^{\text {ini }}$ the initial energy of the double vertex. A derivation of Eq. (4) is given in Appendix B. The magnetizations of the neighbors as well as of the central island are kept uniform during the switching event.

Since only the initial and final state are involved in Eq. (4), we do not need to apply the SISM in this model. In order to keep each island uniformly magnetized and the reversal mechanism a coherent rotation, we consider the energy barrier for one single nanostructure $\Delta E^{\text {isol }}$, which is obtained from a coherent rotation of the magnetization. Thus, this yields $\Delta E^{\text {isol }}=1.37 \mathrm{eV}$.

\section{B. Model 2. Uniform environment}

As a step further, we apply the SISM in this model in such a fashion that the magnetic region $\Omega_{m}$ covers only the central island, where $\Omega_{m}$ denotes the magnetic region, where the SISM iteratively minimizes the energy to find the MEP, taking into account all energy contributions of the system. We continue keeping the $\mathrm{NN}$ islands uniformly magnetized.

Under these assumptions, the total energy of the system also includes the strayfield interactions between the central island and the NN islands, as in the previous model. The interaction energies among the $\mathrm{NN}$ islands are constant during the switching process due to their uniform nature. Therefore, the difference in their saddle point energy and initial energy vanishes. Note that the reversal mechanism is not a perfect coherent rotation anymore. The deviation from the rotation of a perfect uniformly magnetized configuration is associated with the application of the SISM, which allows the relaxation of the magnetization, i.e., deviation from the perfectly uniform configuration, for each step of the MEP taking into account both the shape of the island (shape anisotropy) and the interactions fields originating from the $\mathrm{NN}$ islands.

\section{Model 3. Full micromagnetic model}

For the last approach, we include all the seven islands in the magnetic region $\Omega_{m}$, where the string method is applied. Thus 


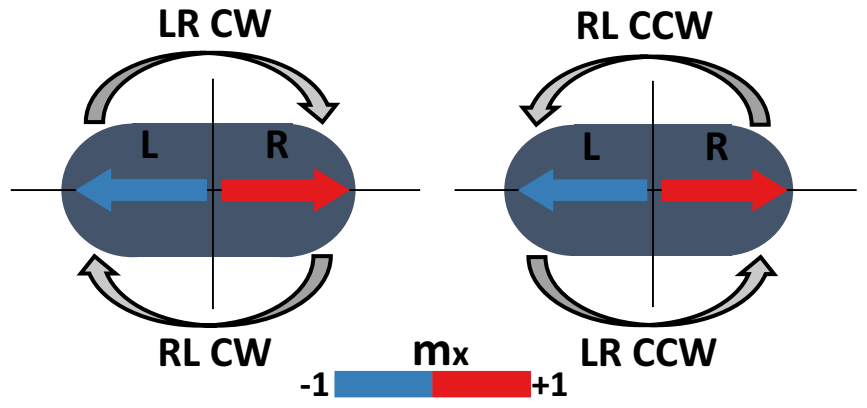

FIG. 3. Rotation directions for the switching event on the central island. We refer to a switching event with $\boldsymbol{m}^{\text {ini }}=(-1,0,0), \boldsymbol{m}_{\mathrm{CCW}}^{\text {saddle }}=$ $(0,-1,0)$, and $\boldsymbol{m}^{\text {fin }}=(1,0,0)$ as a left to right counterclockwise rotation ( $\mathrm{LR} \mathrm{CCW).} \mathrm{If} \mathrm{the} \mathrm{saddle} \mathrm{point} \mathrm{configuration} \mathrm{is} \mathrm{changed} \mathrm{to}$ $\boldsymbol{m}_{\mathrm{CW}}^{\text {saddle }}=(0,1,0)$, this becomes a left to right clockwise (LR CW) rotation. Likewise, one can define a right to left counterclockwise rotation (RL CCW) and a right to left clockwise rotation (RL CW).

both the $\mathrm{NN}$ islands and the central island are dynamically relaxed. The interactions among the $\mathrm{NN}$ islands change at each state of the minimum energy path, so they cannot be neglected in the barrier calculation.

We include indirectly the effect of temperature in our system via reduced $M_{s}$ and $A$ [28-30], allowing for fluctuations to arise from the decreased coupling energy between the spins, which could even form magnetic domains. In Model 3, magnetization inhomogeneities are accounted for not only in the central island but also in the NN islands. Note that Model 2 also takes into account the fluctuations, however only in the magnetization of the central island, since the $\mathrm{NN}$ islands are kept uniform.

\section{RESULTS AND DISCUSSION}

\section{A. Parallel switching channels}

One of the main consequences introduced by Model 2 and Model 3 and that is not predicted by Model 1, is the dependence of the energy barrier on the direction of the rotation of the central island's magnetization. For the sake of simplicity, we give to each rotation direction an acronym, as illustrated in Fig. 3.

Thereby, the switching of the central island's magnetization can occur via two parallel channels, clockwise (CW) and counterclockwise $(\mathrm{CCW})$, which might have a different switching rate depending on the NN configuration. The resulting rate of switching from the Arrhenius law [25,31] is then defined as

$$
f=\frac{1}{\tau}=\frac{1}{\tau_{0}} \cdot\left(e^{-\frac{\Delta E_{\mathrm{CW}}}{k_{B} T}}+e^{-\frac{\Delta E_{\mathrm{CCW}}}{k_{B} T}}\right),
$$

with $\tau$ being the estimated lifetime of the state, $\tau_{0}$ the attempt period, $k_{\mathrm{B}}=8.62 \times 10^{-5} \mathrm{eV} / \mathrm{K}$ the Boltzmann constant and $T$ is the temperature. Note that $\tau_{0}$ might be different for each switching channel. Here, we assume that both channels have the same attempt frequency.
When a mean barrier approximation is used, the switching rate is given as

$$
f^{\text {mean }}=\frac{2}{\tau_{0}} \cdot e^{-\frac{\Delta E^{\text {mean }}}{k_{B} T}},
$$

where $\Delta E^{\text {mean }}$ is the mean barrier given by Eq. (4), which is the average barrier between the $\mathrm{CW}$ and $\mathrm{CCW}$ barriers. However, in the cases where the rates via the $\mathrm{CW}$ and CCW channels are very different, only the fastest channel will dominate. Thereby, the mean-barrier approximation leads to significantly deviating rates underestimating the actual switching rate. A detailed discussion for these deviations is given in Appendix B. Under these assumptions, Eq. (5) can be simplified including only the exponential term corresponding to the faster channel. Hence, the estimated lifetime for a single channel is defined by

$$
\tau=\tau_{0} \cdot e^{\frac{\Delta E}{k_{B} T}} .
$$

To better visualize the physical origin of this splitting, consider the large arrows in Fig. 4 (Model 2) in the snapshots for the saddle point configurations for both $\mathrm{CW}$ and $\mathrm{CCW}$ directions. For the former, one can see that approaching the saddle point configuration, the magnetization vectors of the central island and vertical $\mathrm{NN}$ islands are showing on average in the same direction, leading to an overall poles-attraction configuration. In contrast, during a CCW switching process, the saddle point configuration of the central island points to the opposite direction. Hereby the conflicting magnetic fields give rise to pole repulsion, which yields in an additional energetic level that has to be overcome.

\section{B. Energy barriers}

In order to quantify the effect of parallel switching channels, we first consider a single configuration $c=63$, as illustrated in Fig. 1(c), and analyze the energy barriers for different rotation directions. In this case, a left to right (LR) switching of the magnetization of the central island changes the state of the double vertex from a Type III-Type III double vertex to an energetically favorable Type II-Type II. Figure 5 illustrates the MEPs for both $\mathrm{CW}$ and $\mathrm{CCW}$ rotations, where the initial energies have been shifted to zero (see Table II). The results indicate that the energy barriers for a $\mathrm{CW}$ rotation are lower than for a CCW rotation. As the name suggests, Model 1 is an approximation of the mean barrier (the detailed derivation is given in Appendix B) for the $\mathrm{CW}$ and $\mathrm{CCW}$ rotations, and thus, the energy path from Fig. 5 is obtained as the average energy path for these directions using uniformly magnetized $\mathrm{NN}$ islands and a coherent rotation. Figure 4 illustrates the initial, saddle point and final magnetization configurations for all models. According to Eq. (4), the energy barrier for Model 1 (orange) depends only on energies of the initial and final states and does not distinguish between the rotation directions. Models 2 and 3 (blue and red), however, include the spatial distribution and symmetry of the neighboring islands in the minimization process during the string method, thus breaking this degeneracy.

Namely, when considering the demagnetization field interactions from the neighboring islands, the $\mathrm{CW}$ rotation is energetically favorable over the $\mathrm{CCW}$ rotation since its 


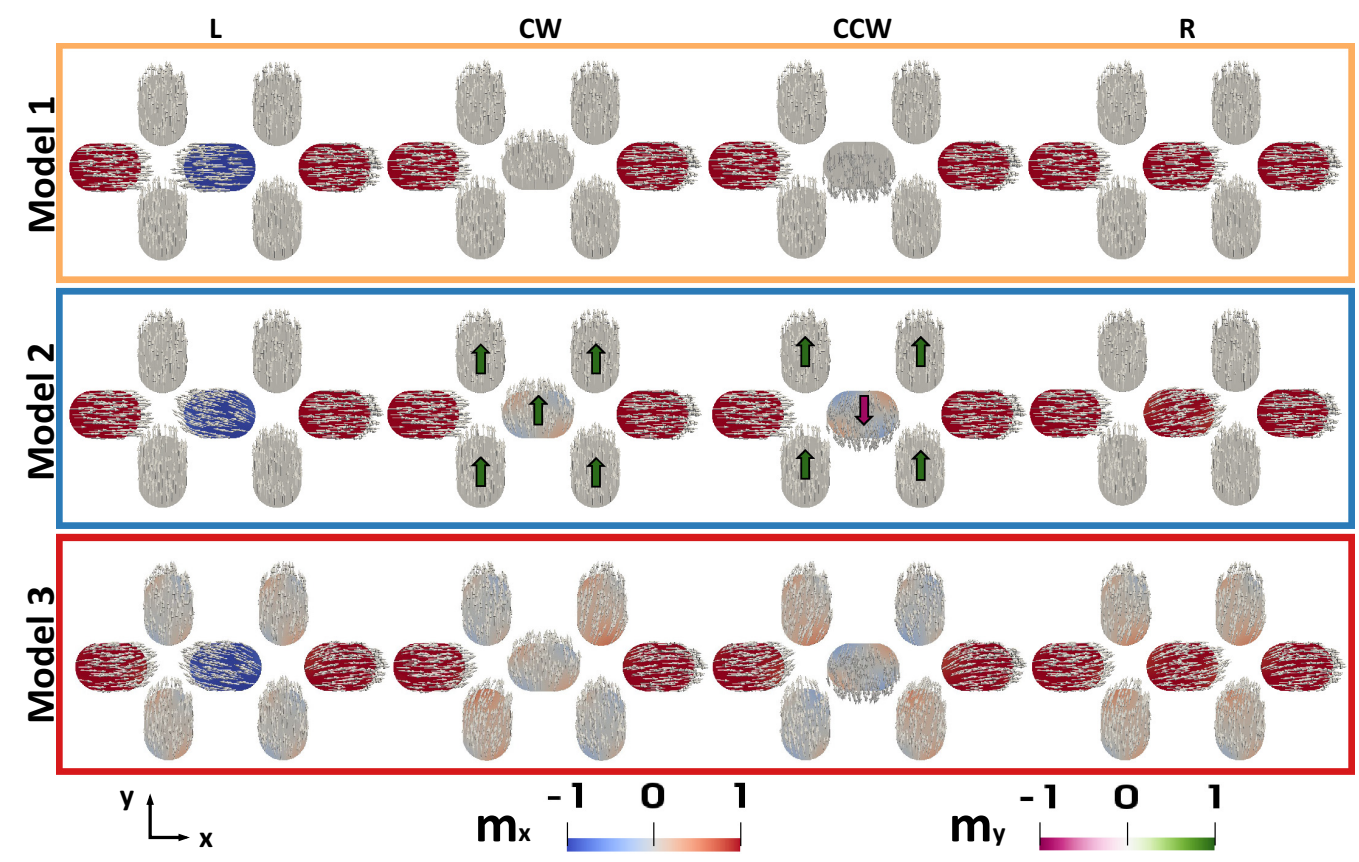

FIG. 4. Snapshots of the magnetization states during the reversal process for configuration $c=63$. Illustration of the $\mathrm{x}$ component of the magnetizations using Model 1 (orange), Model 2 (blue) and Model 3 (red) for the initial state L, saddle point states for both CW and CCW rotation directions and final state $R$. The arrows added in the snapshots (Model 2) indicate average magnetization where the colorbar $m_{y}$ indicates the average $y$ component of the magnetization providing a visual support to understand the magnetostatic interaction between the central island and its NNs.

intermediate magnetization configurations point in positive $y$ direction which aligns with the nonzero $y$ component of the stray field in the configuration $c=63$. Table II shows the values for the energy barriers of the configuration $c=63$ and of the isolated island.

In the following, we focus on the $\mathrm{CW}$ rotations for the configuration $c=63$. All models show a significant reduction of the energy barrier compared to the isolated nanostructure mainly due to the introduction of the stray-field interaction with the NN islands.

In contrast to Model 1, Model 2 introduces nonuniformities in the magnetization of the central island. The reversal mechanism obtained with the SISM, which is not a perfect coherent rotation anymore, is associated to a further reduction of the energy barrier. This discrepancy can be best seen in Fig. 4. Comparing the snapshots at the saddle point configurations, one can see that the Model 2 and Model 3 show deviations from the uniform configuration at the saddle point depicted exemplary in Model 1 and a nonvanishing $x$ component of the magnetization develops at the saddle point (in Model 3 also in the vertical NN islands), at variance with the coherent rotation assumption made in Model 1.

Compared to the widely used mean-barrier approximation (Model 1), Model 2 reduces the lowest energy barrier (LR CW) about $28 \%$.

The highest energy barrier reduction is observed for Model 3 , which is the full micromagnetic model. In addition to Model 2, the neighbors are included in the magnetic region of the SISM, where the energy is minimized. Thus their magnetizations are dynamically relaxed and may change for each intermediate state in the transition path.

In this case, the stray-field interactions among the neighboring islands are not constant and the difference of their saddle point and initial states energies does not vanish. The lowest energy barrier for this model is reduced by $33 \%$ compared to Model 1 for the configuration $c=63$.

Furthermore, only for Model 3 the magnetic system is in a true energetic equilibrium, since all islands are added in the

TABLE II. Energy barriers and estimated switching times obtained by applying the Models 1-3. The numerical results of the switching barriers $\Delta E$ and average lifetimes $\tau$ to switch the central island's magnetization from left to right (LR) for both CW and CCW rotation directions, as visualized in Fig. 3. The estimated switching times were calculated with Eq. (7) using $\tau_{0}=10^{-10} \mathrm{~s}$ and $T=300 \mathrm{~K}$.

\begin{tabular}{|c|c|c|c|c|c|c|}
\hline \multirow{3}{*}{$\begin{array}{l}\text { Direction } \\
\text { MODEL No. }\end{array}$} & \multirow{3}{*}{$\begin{array}{c}\text { Isolated } \\
-\end{array}$} & \multicolumn{5}{|c|}{ Configuration $c=63$} \\
\hline & & \multirow{2}{*}{$\frac{\mathrm{CCW} / \mathrm{CW}}{1}$} & \multicolumn{2}{|c|}{ LR CCW } & \multicolumn{2}{|c|}{ LR CW } \\
\hline & & & 2 & 3 & 2 & 3 \\
\hline$\Delta E(\mathrm{eV})$ & 1.37 & 1.16 & 1.51 & 1.37 & 0.83 & 0.77 \\
\hline$\tau(\mathrm{s})$ & $9.8 \times 10^{12}$ & $2.4 \times 10^{9}$ & $2.9 \times 10^{15}$ & $1.2 \times 10^{13}$ & $7.2 \times 10^{3}$ & $8.4 \times 10^{2}$ \\
\hline
\end{tabular}




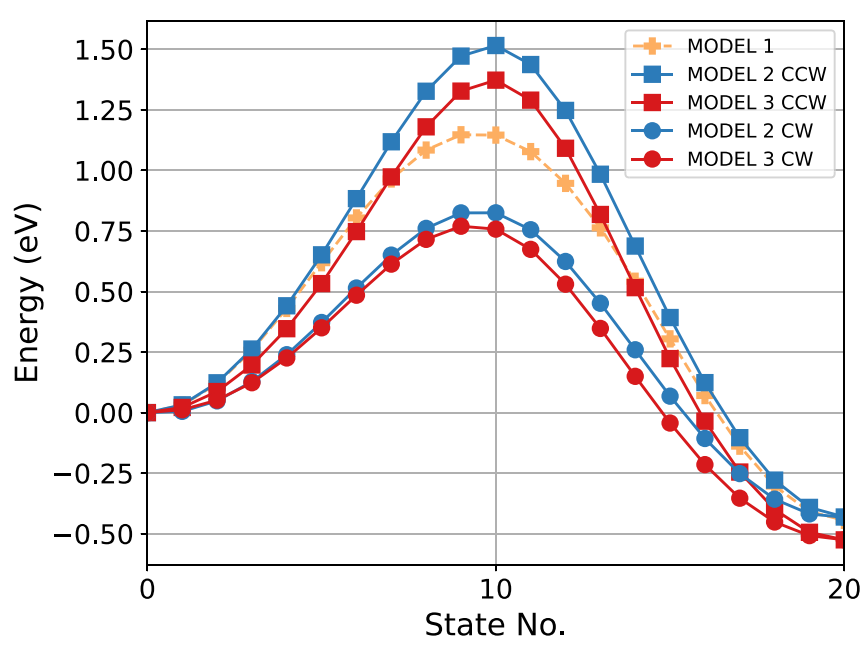

FIG. 5. Minimum energy paths for the switching of the magnetization of the central island. The MEPs for $c=63$ considering both $\mathrm{CCW}$ (squares) and CW (circles) rotations, where the initial energies are shifted to zero. Here, the initial double vertex contains two Type III vertices and one obtains two Type II vertices after switching the magnetization of the central island from $\boldsymbol{m}^{\text {ini }}=(-1,0,0)$ to $\boldsymbol{m}^{\mathrm{fin}}=(1,0,0)$, represented by State No. $=0$ and State No. $=20$, respectively.

magnetic region of the string method and their energies have been minimized.

Animations of the switching process for the configuration $c=63$ using Models 2 and 3 can be found in the Supplemental Material [32].

Although the differences between the Models 2 and 3 may seem rather small, they have a major impact on the average lifetime given by Eq. (7). Since the fastest channel dominates the switching, we compare the estimated switching times for LR CW rotation directions for the configuration $c=63$.

Here we use the attempt period $\tau_{0}=10^{-10} \mathrm{~s}$ and $T=$ $300 \mathrm{~K}$.

The value of the attempt period is only exemplary to show the role of the energy barrier reduction regarding the switching times. The values for $\tau$ are given in Table II.

One can see that the estimated lifetime (LR CW) calculated using Model 2 is approximately up to six orders of magnitudes lower compared to Model 1, and one order of magnitude higher with respect to Model 3.

The full micromagnetic model yields a reduction of the average lifetime by about seven orders of magnitude with respect to the average barrier Model 1, often used in ASIs literature.

To show that Model 3 predicts (in nearly all cases) the lowest energy barriers, we calculate the switching barriers for all possible configurations $c$ in a double vertex given by Eq. (2).

Figure 6 shows a direct comparison between the models for both LR CCW and LR CW rotations.

To obtain the barriers with respect to the final state (RL) one has to rotate the islands $180^{\circ}$ around both the vertical and horizontal axis, since the rotation direction also changes. In principle, there exists an equivalent $\mathrm{CCW}$ barrier for each $\mathrm{CW}$ barrier, but with a different configuration number. If we
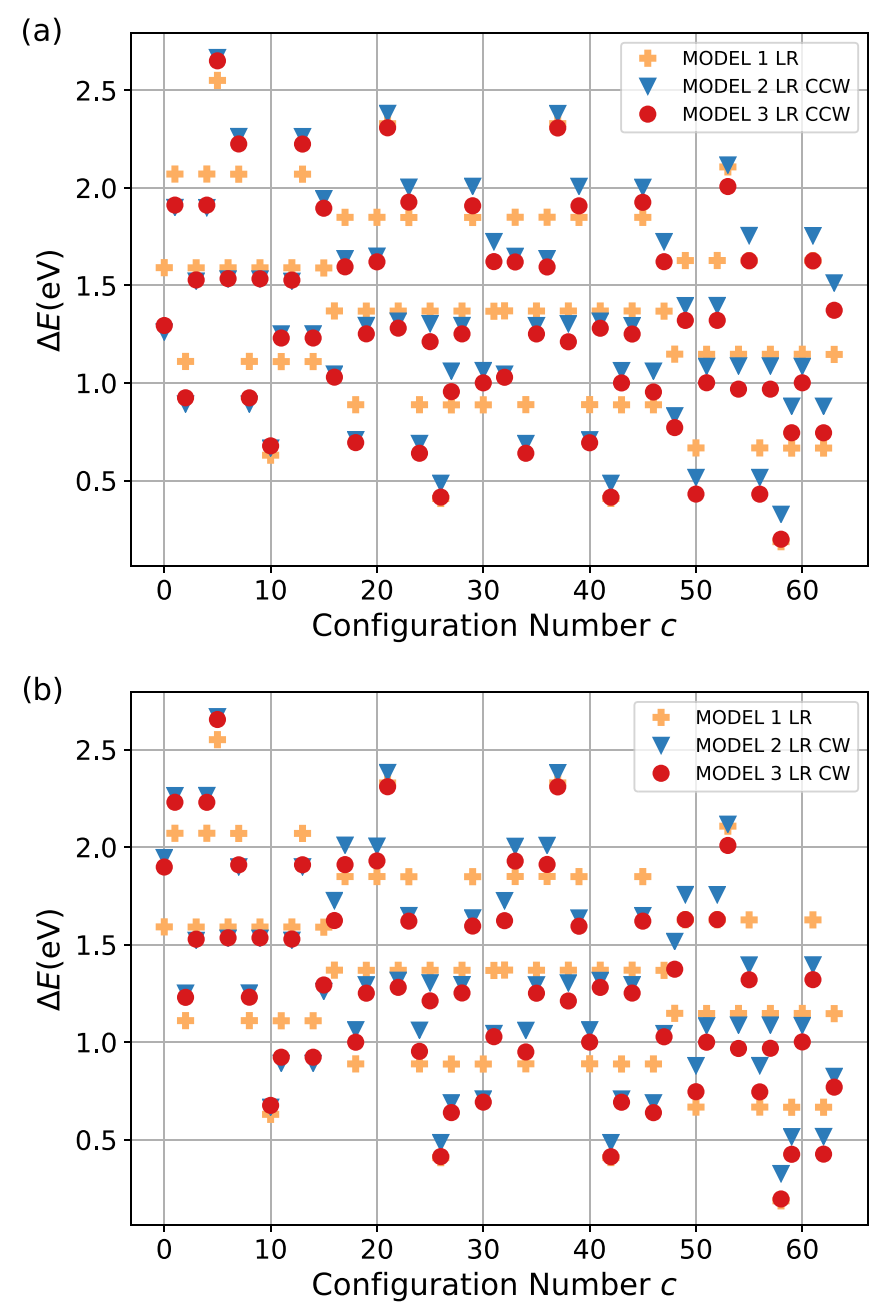

FIG. 6. Direct comparison between the Models. Energy barriers for the switching of the magnetization of the central island using all possible configurations of the $\mathrm{NN}$ islands in a double vertex illustrated in Fig. 1(c) via LR CCW (a) and LR CW (b) rotation directions.

consider $c=63$ as an example, $c=0 \mathrm{LR} \mathrm{CCW}$ has the same barrier as $c=63 \mathrm{RL} \mathrm{CCW}$.

Figure 6 shows that for all configurations at least one of the possible energy barriers is the lowest for Model 3.

Since Model 3 describes the evolution of the system through a saddle point configuration, where each $\mathrm{NN}$ island is dynamically relaxed to its energetic equilibrium, it yields the most realistic barrier, whereby the initial and final states are true energy minima. Note that Model 1, as well as Model 2, can result, in some cases, in lower energy barriers compared to Model 3. As it can be seen in Fig. 4, the magnetization of the NN islands are kept uniform for the first two models. Hereby, the energies of the initial and final states are not properly minimized, and thus the systems have not reached their true energetic equilibrium. This points out further limitations of these models. Consider that nonuniformities in the central island's magnetization modeled by Model 2 reduces the initial and final state energies $E^{\text {ini }}$ and $E^{\text {fin }}$, respectively as the system approaches its energetic equilibrium by a certain amount $\Delta_{i, 2}$ with respect to the uniform states utilized in Model 1. The 


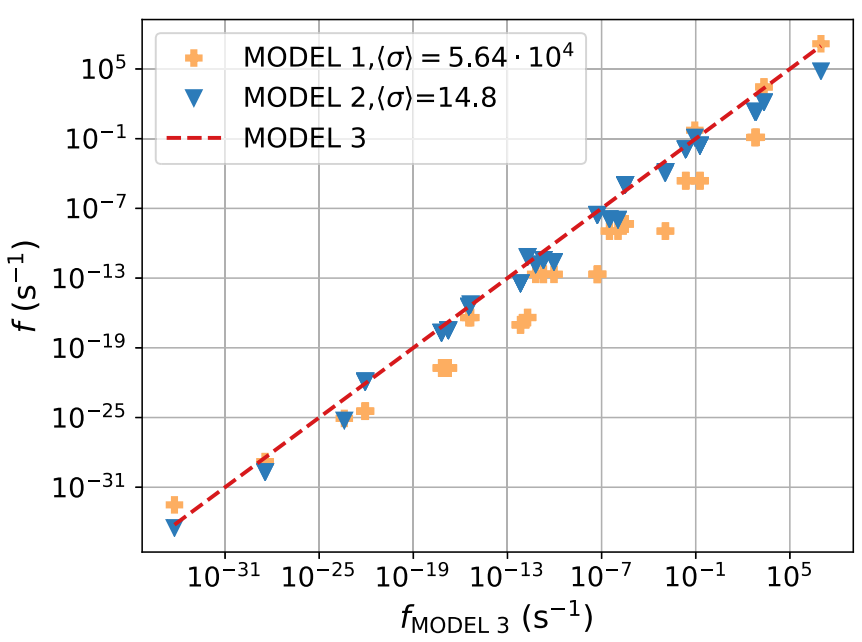

FIG. 7. Switching rates for Models 1-3. Log-log plot of the switching rates given by Eq. (5) for each model. The $x$ axis shows the switching rates for the possible 64 configurations using Model 3 , whereas the $y$ axis shows the switching rates for the Models 1 (orange, crosses) and 2 (blue, triangles). The mean error $\langle\sigma\rangle_{\text {Model }_{\mathrm{i}}}$, where $i=1$ or $i=2$, given by Eq. (8), is shown in the legend. The values of Model 3 are plotted as a reference line.

saddle point energy $E^{\text {saddle }}$ is assumed to be reduced by $\Delta_{s, 2}$. If $\Delta_{s, 2}>\Delta_{i, 2}$ the switching barrier calculated with Model 2 is lower (for a given rotation direction). However, only considering Model 3 the micromagnetic simulations can be performed to their full extent to reach the overall equilibrium states. As a consequence, the reduction of the initial and final state energies, $\Delta_{i, 3}$ for the full micromagnetic model is always greater than $\Delta_{i, 2}$. Hence, an equal reduction of the saddle point energies for both Models 2 and 3, and thus, $\Delta_{s, 2}=\Delta_{s, 3}$ can lead to a lower energy barrier for Model 2, since $\Delta_{i, 3}>\Delta_{i, 2}$.

\section{Switching rates}

Using our results from Sec. III B, the corresponding switching rates are calculated with Eqs. (5) and (6), where the correlation between the models is illustrated in Fig. 7.

The mean error

$$
\langle\sigma\rangle_{\text {Model }_{\mathrm{i}}}=\frac{1}{64} \sum_{c} \frac{f_{\mathrm{Model}_{3}, c}}{f_{\mathrm{Model}_{\mathrm{i}}, c}},
$$

where the value $\sum_{c} \frac{f_{\text {Model }, c}}{f_{\text {Model }, c}, c}$ sums over the relative deviation factors for all configurations $c$, and gives an estimate of the average deviation from the full micromagnetic model. The higher the deviation from. $\langle\sigma\rangle=1$, the more inaccurate are the calculated energy barriers compared to the full micromagnetic model, Model 3.

Even though Model 1 might be a mathematically valid approximation of the mean-barrier based on the derivation given in Appendix B, the concept of an average barrier does not necessarily imply a physically justifiable input for the kinetic Monte Carlo simulations, as the mean-barrier approximation approaches its limitations, for significantly different energy barriers for CCW and CW channels. As shown by the mean error $\langle\sigma\rangle_{\text {Model }_{1}}=5.64 \times 10^{4}$, the mean switching rate is

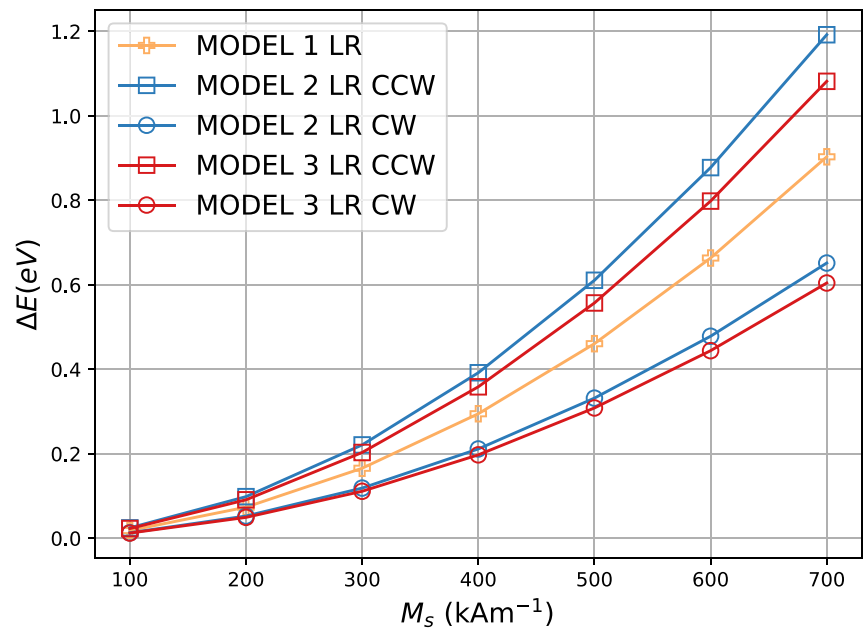

FIG. 8. Dependence of energy barriers on sASI material parameters for configuration $c=63$. Calculated switching barriers as a function of the saturation magnetization $M_{s}$ using Model 1 (orange, crosses), Model 2 (blue), and Model 3 (red) for both LR CCW (squares) and LR CW (circles) rotation directions.

underestimated by over four orders of magnitudes compared to the full micromagnetic model. With the introduction of parallel switching channels and the nonuniformities in the central island's magnetization in Model 2, the mean switching rate already improves significantly compared to Model 1, and the mean error $\langle\sigma\rangle_{\text {Model }_{2}}=15.4$ decreases three orders of magnitude. Nevertheless, compared to the full micromagnetic model, the switching rates are underestimated in general and even overestimated for the cases this model reaches its limitations, as explained in Sec. IV B.

Considering once more the configuration $c=63$, we calculate the exact average barrier using the values obtained from Model 3, hence, the average barrier is $\Delta E^{\text {avg }}=$ $1 / 2(0.77+1.37) \mathrm{eV}=1.07 \mathrm{eV}$. For the switching rates of the configuration $c=63$ calculated with Eqs. (5) and (6) we obtain $f_{\text {Model }_{3}}=1.2 \times 10^{-3} \mathrm{~s}^{-1}$ and $f^{\text {avg }}=2.2 \times 10^{-8} \mathrm{~s}^{-1}$. Compared to $f^{\text {mean }}$ obtained with the mean-barrier from Model 1, which is $f_{\text {Model }_{1}}=6.6 \times 10^{-10} \mathrm{~s}^{-1}$, we see that the proper micromagnetic modeling $f_{\text {Model }_{1}} \rightarrow f^{\text {avg }}$ leads to an increase of the switching rate up to two orders of magnitudes, whereas differentiation between parallel switching channels $f^{\text {avg }} \rightarrow f_{\mathrm{Model}_{3}}$ improves the switching rate by additional five orders of magnitude.

\section{Material parameters}

In order to further validate our findings, we vary the saturation magnetization $M_{s}$ and calculate the energy barriers for a LR switching using the configuration $c=63$. Here the exchange stiffness constant is scaled $[29,30,33]$ according to $A\left(M_{s}\right)=A_{0} \cdot\left(M_{s} / M_{0}\right)^{1.7}$, where $A_{0}=18 \mathrm{pJ} / \mathrm{m}$ and $M_{0}=$ $950 \mathrm{kA} / \mathrm{m}$ are the exchange stiffness and saturation magnetization at $T=0 \mathrm{~K}$.

As depicted in Fig. 8, independently from the choice of the material parameters, the full micromagnetic model gives always the lowest energy barriers, and thus, the highest switching rates. Furthermore, the differences between the $\mathrm{CW}$ 
and CCW switching channels increase with $M_{s}$, yet the CW channel yields always the lower barrier. Note that a decrease in $M_{s}$ is equivalent to an increase of the temperature at which the simulations are performed. Hence, as $M_{s}$ decreases the temperature of the system approaches $T_{\text {Curie }}$ and the overall barriers are decreasing, so significantly diminishing the absolute differences between the models. That being said, in order to effectively calculate energy barriers and reproduce experiments, it is of crucial importance to accurately determine the material laws for $A$ and $M_{s}$ as a function of temperature. The scaling method can be seen as a limitation of the proposed full micromagnetic model, where the effect of thermal fluctuations is introduced only indirectly via the above mentioned micromagnetic parameters, rather than making use of stochastic methods like Langevin dynamics [34,35], Landau-LifshitzBloch equation [36-38] or constrained Monte Carlo [39].

In summary, our results indicate that the values obtained with Model 1 deviate significantly from the values of Model 3 . The mean barrier approximation falsely overestimates the energy barriers when an interaction field with a nonvanishing $y$ component acts on the central island, and thus, the $\mathrm{CW}$ and $\mathrm{CCW}$ barriers are different. As a consequence, the switching rates are underestimated. Although Model 2 recovers the most important shortcomings of Model 1 by using micromagnetics and differentiating between $\mathrm{CW}$ and $\mathrm{CCW}$ channels, it is Model 3 that gives the lowest and more realistic energy barriers. Thereby, independently from the chosen material parameters, Model 3 provides the best approximation of the energy barriers and transition rates that should be utilized to model dynamical processes in sASI lattices, since the probability of switching the magnetization of an island is directly proportional to these rates.

\section{CONCLUSIONS}

In this paper, we use the SISM to calculate all energy barriers to switch the magnetization of the central island of a double vertex in square artificial spin ice.

We investigate the influence of the nearest-neighbor islands on the energy barrier in a square artificial spin ice lattice by calculating the energy barrier with three different approaches.

In the first model we consider the widely used mean-barrier approximation. Besides the nonuniformities in the magnetization of the island of interest already considered in Model 2, the last model, Model 3, is a full micromagnetic model where each magnetization is dynamically relaxed depending on each other.

As a first relevant result, Model 2 yields different minimum energy paths for CCW and CW rotation directions for particular configurations, where the most probable switching occurs via the channel with the lowest energy barrier. This distinction between $\mathrm{CW}$ and $\mathrm{CCW}$ reversal is completely neglected by the average model, Model 1, often utilized in ASIs literature.

To conclude our results, the energy barrier for switching the magnetization of an island in an artificial spin ice lattice can be reduced significantly applying a full micromagnetic model on a double vertex (Model 3) compared to the energy barrier obtained with the mean-barrier approximation.

The interactions originating from the demagnetization fields from the neighboring islands and the consequent energy difference between $\mathrm{CW}$ and $\mathrm{CCW}$ reversal paths are the first key reason for this reduction. The inhomogeneities in the magnetizations of both the central islands and of the dynamically relaxed neighbors, that arise during the reversal, introduce an additional contribution to the reduction of the energy barriers. Both effects are neglected in the mean-barrier approximation.

In most drastic cases the mean-barrier approximation can result in energy barriers 35\% higher as Model 3.

While the ad hoc reduction of the barriers often applied in mean-barrier model can fix particular barriers, it can overor underestimate the barriers leading to different dynamics when simulating artificial spin ice systems. The main reason resides in the fact that an average barrier reaches its limitations and has no physical significance regarding the switching rates utilized in the kinetic Monte Carlo simulations.

The presented full micromagnetic model is general and can also be applied for other artificial spin ice lattice types and magnetic materials, if both the number and spatial arrangement of the nearest neighbors are adapted.

\section{ACKNOWLEDGMENTS}

The computational results presented have been achieved [in part] using the Vienna Scientific Cluster (VSC). S.K., C.A., C.V., F.S., F.B., P.H., and D.S. gratefully acknowledge the financial support by the Austrian Federal Ministry for Digital and Economic Affairs and the National Foundation for Research, Technology and Development. N.L., M.M., and P.V. acknowledge support from the Spanish Ministry of Economy, Industry and Competitiveness under the Maria de Maeztu Units of Excellence Programme - MDM-2016-0618 and the project RTI2018-094881-B-I00 (MINECO/FEDER). N.L. has received funding from the European Union's Horizon 2020 research and innovation programme under the Marie Słodowska Curie Grant Agreement No. 844304 (LICONAMCO). K.H. acknowledges funding by the Swiss National Science Foundation (Project No. 200020_172774).

\section{APPENDIX A: MICROMAGNETIC ENERGIES}

According to the SISM, we minimize the total energy of a chosen magnetic region $\Omega_{m}$. For our purposes, we consider the total energy by means of micromagnetics [40] as

$$
E^{\mathrm{tot}}=E^{\mathrm{dem}}+E^{\mathrm{ex}},
$$

where $E^{\text {dem }}$ denotes the demagnetization energy and $E^{\text {ex }}$ represents the ferromagnetic exchange energy. We do not consider any externally applied magnetic fields. The demagnetization energy $E^{\mathrm{dem}}$ is described as

$$
E^{\mathrm{dem}}=-\frac{\mu_{0} M_{s}}{2} \int_{\Omega_{m}} \boldsymbol{m} \cdot \boldsymbol{H}^{\mathrm{dem}} \mathrm{d} \boldsymbol{x},
$$

where $\mu_{0}$ is the vacuum permeability, $\Omega_{m}$ defines the magnetic region, and $\boldsymbol{m}$ represents the normalized magnetization vector.

The demagnetizing field $\boldsymbol{H}^{\mathrm{dem}}$ is given by

$$
\boldsymbol{H}^{\mathrm{dem}}(\boldsymbol{x})=-\frac{M_{\mathrm{s}}}{4 \pi} \int_{\Omega_{\mathrm{m}}} \nabla \nabla^{\prime} \frac{1}{\left|\boldsymbol{x}-\boldsymbol{x}^{\prime}\right|} \boldsymbol{m}\left(\boldsymbol{x}^{\prime}\right) \mathrm{d} \boldsymbol{x}^{\prime} .
$$


Moreover, the ferromagnetic exchange energy favoring a parallel alignment of the spins is defined as

$$
E^{\mathrm{ex}}=\int_{\Omega_{m}} A(\nabla \boldsymbol{m})^{2} \mathrm{~d} \boldsymbol{x},
$$

where $A$ is the exchange stiffness constant.

\section{APPENDIX B: MEAN-BARRIER APPROXIMATION}

Especially for kinetic Monte Carlo simulations of artificial spin ice, the mean switching barrier is often expressed solely depending on the energies of the initial and final energy state. The initial and final state energies can be easily calculated using the point-dipole model or regular micromagnetic simulation codes, where each island is uniformly magnetized, with a single-island switching barrier $E^{\text {isol }}$ added as an independent parameter.

The reason that the energies of the intermediate states do not need to be considered in this simple approximation is based on the symmetry properties of the stray field and Zeeman interactions, whose energies are antisymmetric under rotations of $\pi$, i.e., $E(\phi+\pi)=-E(\phi)$. Under the assumptions of a Stoner-Wohlfarth particle as central island and constantly magnetized neighboring islands, the total energy of the central island is given by an anisotropy and Zeeman term in the form

$$
E(\boldsymbol{m}, \boldsymbol{H})=E^{\mathrm{ani}}(\boldsymbol{m})+E^{\mathrm{zee}}(\boldsymbol{m}, \boldsymbol{H}),
$$

with

$$
\begin{gathered}
E^{\text {ani }}(\boldsymbol{m})=-K_{V}(\boldsymbol{m} \cdot(1,0,0))^{2}, \\
E^{\mathrm{zee}}(\boldsymbol{m}, \boldsymbol{H})=-\mu_{0} \mu_{s} \boldsymbol{m} \cdot \boldsymbol{H},
\end{gathered}
$$

where $K_{V}=k_{\text {eff }} \cdot V$ denotes the product of the effective anisotropy constant $k_{\text {eff }}$ (basically due to the shape anisotropy) and the volume of the isolated nanostructure $V$, and $\mu_{s}=$ $M_{s} \cdot V$ is the total magnetic moment. Furthermore, we assume that this field is sufficiently weak, so that both the initial and final states can be approximated by $\boldsymbol{m}^{\text {ini }}=(-1,0,0)$ and $\boldsymbol{m}^{\text {fin }}=(1,0,0)$. In general, the value of the activation barrier is given by the difference between the saddle point energy $E^{\text {saddle }}$ as given by Eq. (1), i.e., central moment pointing up, $E_{\mathrm{CW}}^{\text {saddle }}$ with $\boldsymbol{m}_{\mathrm{CW}}^{\text {saddle }}=(0,1,0)$, or down, $E_{\mathrm{CCW}}^{\text {saddle }}$ with $\boldsymbol{m}_{\mathrm{CCW}}^{\text {saddle }}=$ $(0,-1,0)$ in a configuration $c$, and the initial energy of the configuration $E^{\text {ini }}$. Note, that the magnetization state with the maximum energy involved in the energy barrier calculation can significantly deviate from $\boldsymbol{m}_{\mathrm{CW} / \mathrm{CCW}}^{\text {saddle }}$ for high interaction fields. We assume that this deviation is also negligible. In this context we obtain

$$
\begin{aligned}
& E^{\mathrm{ini}}(\boldsymbol{H})=E\left(\boldsymbol{m}^{\mathrm{ini}}, \boldsymbol{H}\right)=-K_{V}+\mu_{0} \mu_{s} H_{x}, \\
& E^{\mathrm{fin}}(\boldsymbol{H})=E\left(\boldsymbol{m}^{\mathrm{fin}}, \boldsymbol{H}\right)=-K_{V}-\mu_{0} \mu_{s} H_{x}, \\
& E_{\mathrm{CW}}^{\text {saddle }}(\boldsymbol{H})=E\left(\boldsymbol{m}_{\mathrm{CW}}^{\text {saddle }}, \boldsymbol{H}\right)=-\mu_{0} \mu_{s} H_{y}, \\
& E_{\mathrm{CCW}}^{\text {saddle }}(\boldsymbol{H})=E\left(\boldsymbol{m}_{\mathrm{CCW}}^{\text {saddle }}, \boldsymbol{H}\right)=+\mu_{0} \mu_{s} H_{y} .
\end{aligned}
$$

We get for the energy barrier of an isolated nanostructure

$$
\Delta E^{\text {isol }}=E_{\mathrm{CW}}^{\text {saddle }}(\boldsymbol{H}=(0,0,0))-E^{\mathrm{ini}}(\boldsymbol{H}=(0,0,0))=K_{V} \text {. }
$$

With this result, we are finally able to express the meanenergy barrier of the $\mathrm{CW}$ and CCW channels by $\Delta E^{\text {isol }}, E^{\text {fin }}$, and $E^{\text {fin }}$ as in Eq. (4):

$$
\begin{aligned}
\Delta E^{\text {mean }} & =1 / 2\left(\Delta E_{\mathrm{CW}}^{\text {saddle }}+\Delta E_{\mathrm{CCW}}^{\text {saddle }}\right) \\
& =1 / 2\left(E_{\mathrm{CW}}^{\mathrm{saddle}}-E^{\mathrm{ini}}+E_{\mathrm{CCW}}^{\mathrm{saddle}}-E^{\mathrm{ini}}\right) \\
& =K_{V}-\mu_{0} \mu_{s} H_{x} \\
& =\Delta E^{\mathrm{isol}}+1 / 2\left(E^{\mathrm{fin}}-E^{\mathrm{ini}}\right) .
\end{aligned}
$$

Based on the aforementioned assumptions, Eq. (4) is an approximation of an average barrier between the two energy barriers for the corresponding parallel switching channels. Even when the initial and final states are involved in this equation, it might neglect the interaction fields originating from the neighboring islands. In a case, where $E^{\text {ini }}=E^{\text {fin }}$, the mean-barrier coincides with the energy barrier of one isolated nanostructure, e.g., configuration $c=31$. But in reality there is still an effective field acting on the central island. According to the Stoner-Wohlfarth Model, the energy barrier of switching the magnetization of a single domain particle under the influence of an external field $\boldsymbol{H}=\left(0, H_{y}, 0\right)$ is $\Delta E=\Delta E^{\text {isol }}\left(1-\frac{H_{y}}{H_{k}}\right)^{2}$, where $H_{k}$ is the strength of the anisotropy field and $H_{y}$ the strength of the field acting on the single-domained particle [41]. With Eq. (4) one would obtain that $\Delta E^{\text {mean }}=\Delta E^{\text {isol }}$. This deviation points out once more, that the mean-barrier approximation method needs further corrections, to be used as input for kinetic Monte Carlo simulations.
[1] R. F. Wang, C. Nisoli, R. S. Freitas, J. Li, W. McConville, B. J. Cooley, M. S. Lund, N. Samarth, C. Leighton, V. H. Crespi, and P. Schiffer, Artificial 'spin ice' in a geometrically frustrated lattice of nanoscale ferromagnetic islands, Nature (London) 439, 303 (2006).

[2] C. Nisoli, R. Moessner, and P. Schiffer, Colloquium: Artificial spin ice: Designing and imaging magnetic frustration, Rev. Mod. Phys. 85, 1473 (2013).
[3] L. J. Heyderman and R. L. Stamps, Artificial ferroic systems: Novel functionality from structure, interactions and dynamics, J. Condens.: Matter Phys. 25, 363201 (2013).

[4] S. H. Skjærvø, C. H. Marrows, R. L. Stamps, and L. J. Heyderman, Advances in artificial spin ice, Nat. Rev. Phys. 2, 13 (2020).

[5] C. Nisoli, V. Kapaklis, and P. Schiffer, Deliberate exotic magnetism via frustration and topology, Nat. Phys. 13, 200 (2017). 
[6] E. Östman, U. B. Arnalds, V. Kapaklis, A. Taroni, and B. Hjörvarsson, Ising-like behavior of mesoscopic magnetic chains, J. Condens.: Matter Phys. 30, 365301 (2018).

[7] V. Kapaklis, U. B. Arnalds, A. Farhan, R. V. Chopdekar, A. Balan, A. Scholl, L. J. Heyderman, and B. Hjörvarsson, Thermal fluctuations in artificial spin ice, Nat. Nanotechnol. 9, 514 (2014).

[8] G. Möller and R. Moessner, Artificial Square Ice and Related Dipolar Nanoarrays, Phys. Rev. Lett. 96, 237202 (2006).

[9] A. Remhof, A. Schumann, A. Westphalen, H. Zabel, N. Mikuszeit, E. Y. Vedmedenko, T. Last, and U. Kunze, Magnetostatic interactions on a square lattice, Phys. Rev. B 77, 134409 (2008).

[10] V. Kapaklis, U. B. Arnalds, A. Harman-Clarke, E. T. Papaioannou, M. Karimipour, P. Korelis, A. Taroni, P. C. W. Holdsworth, S. T. Bramwell, and B. Hjörvarsson, Melting artificial spin ice, New J. Phys. 14, 035009 (2012).

[11] Z. Budrikis, K. L. Livesey, J. P. Morgan, J. Akerman, A. Stein, S. Langridge, C. H. Marrows, and R. L. Stamps, Domain dynamics and fluctuations in artificial square ice at finite temperatures, New J. Phys. 14, 035014 (2012).

[12] S. John Greaves and H. Muraoka, Formation of thermally induced ground states in two-dimensional square spin ices, J. Appl. Phys. 112, 043909 (2012).

[13] S. Zhang, I. Gilbert, C. Nisoli, G.-W. Chern, M. J. Erickson, L. O'Brien, C. Leighton, P. E. Lammert, V. H. Crespi, and P. Schiffer, Crystallites of magnetic charges in artificial spin ice, Nature (London) 500, 553 (2013).

[14] J. M. Porro, A. Bedoya-Pinto, A. Berger, and P. Vavassori, Exploring thermally induced states in square artificial spin-ice arrays, New J. Phys. 15, 055012 (2013).

[15] A. Farhan, P. M. Derlet, A. Kleibert, A. Balan, R. V. Chopdekar, M. Wyss, J. Perron, A. Scholl, F. Nolting, and L. J. Heyderman, Direct Observation of Thermal Relaxation in Artificial Spin Ice, Phys. Rev. Lett. 111, 057204 (2013).

[16] A. B. Bortz, M. H. Kalos, and J. L. Lebowitz, A new algorithm for Monte Carlo simulation of ising spin systems, J. Comput. Phys. 17, 10 (1975).

[17] S. H. Charap, P.-L. Lu, and Y. He, Thermal stability of recorded information at high densities, IEEE Trans. Magn. 33, 978 (1997).

[18] D. Thonig, S. Reißaus, I. Mertig, and J. Henk, Thermal string excitations in artificial spin-ice square dipolar arrays, J. Condens. Matter Phys. 26, 266006 (2014).

[19] A. Farhan, P. M. Derlet, A. Kleibert, A. Balan, R. V. Chopdekar, M. Wyss, L. Anghinolfi, F. Nolting, and L. J. Heyderman, Exploring hyper-cubic energy landscapes in thermally active finite artificial spin-ice systems, Nat. Phys. 9, 375 (2013)

[20] A. Farhan, P. M. Derlet, L. Anghinolfi, A. Kleibert, and L. J. Heyderman, Magnetic charge and moment dynamics in artificial kagome spin ice, Phys. Rev. B 96, 064409 (2017).

[21] H. Arava, N. Leo, D. Schildknecht, J. Cui, J. Vijayakumar, P. M. Derlet, A. Kleibert, and L. J. Heyderman, Engineering relaxation pathways in building blocks of artificial spin ice for computation, Phys. Rev. Appl. 11, 054086 (2019).

[22] X. Zhang, Y. Lao, J. Sklenar, N. S. Bingham, J. T. Batley, J. D. Watts, C. Nisoli, C. Leighton, and P. Schiffer, Understanding thermal annealing of artificial spin ice, APL Mater. 7, 111112 (2019).

[23] W. E, W. Ren, and E. Vanden-Eijnden, Simplified and improved string method for computing the minimum energy paths in barrier-crossing events, J. Chem. Phys. 126, 164103 (2007).

[24] E. Wigner, The transition state method, Trans. Faraday Soc. 34, 29 (1938).

[25] W. T. Coffey and Y. P. Kalmykov, Thermal fluctuations of magnetic nanoparticles: Fifty years after brown, J. Appl. Phys. 112, 121301 (2012).

[26] C. Abert, L. Exl, F. Bruckner, A. Drews, and D. Suess, magnum.fe: A micromagnetic finite-element simulation code based on fenics, J. Magn. Magn. Mater. 345, 29 (2013).

[27] C. Geuzaine and J.-F. Remacle, Gmsh: A 3d finite element mesh generator with built-in pre- and post-processing facilities, Int. J. Numer. Meth. Eng. 79, 1309 (2009).

[28] F. Heider and W. Williams, Note on temperature dependence of exchange constant in magnetite, Geophys. Res. Lett. 15, 184 (1988).

[29] E. Martinez, L. Lopez-Diaz, L. Torres, and O. Alejos, Temperature dependence of spontaneous magnetization using a continuous model, IEEE Trans. Magn. 39, 2522 (2003).

[30] R. Moreno, R. F. L. Evans, S. Khmelevskyi, M. C. Muñoz, R. W. Chantrell, and O. Chubykalo-Fesenko, Temperaturedependent exchange stiffness and domain wall width in co, Phys. Rev. B 94, 104433 (2016).

[31] W. F. Brown, Thermal fluctuations of a single-domain particle, Phys. Rev. 130, 1677 (1963).

[32] See Supplemental Material at http://link.aps.org/supplemental/ 10.1103/PhysRevB.102.064410 for animations of switching processes using Models 2 and 3.

[33] K. Niitsu, Temperature dependence of magnetic exchange stiffness in iron and nickel, J. Phys. D 53, 39LT01 (2020).

[34] T. Schrefl, W. Scholz, D. Suss, and J. Fidler, Langevin micromagnetics of recording media using subgrain discretization, IEEE Trans. Magn. 36, 3189 (2000).

[35] W. Scholz, T. Schrefl, and J. Fidler, Micromagnetic simulation of thermally activated switching in fine particles, J. Magn. Magn. Mater. 233, 296 (2001).

[36] U. Atxitia, O. Chubykalo-Fesenko, N. Kazantseva, D. Hinzke, U. Nowak, and R. W. Chantrell, Micromagnetic modeling of laser-induced magnetization dynamics using the LandauLifshitz-Bloch equation, Appl. Phys. Lett. 91, 232507 (2007).

[37] R. F. L. Evans, D. Hinzke, U. Atxitia, U. Nowak, R. W. Chantrell, and O. Chubykalo-Fesenko, Stochastic form of the Landau-Lifshitz-Bloch equation, Phys. Rev. B 85, 014433 (2012).

[38] C. Vogler, C. Abert, F. Bruckner, and D. Suess, LandauLifshitz-Bloch equation for exchange-coupled grains, Phys. Rev. B 90, 214431 (2014).

[39] P. Asselin, R. F. L. Evans, J. Barker, R. W. Chantrell, R. Yanes, O. Chubykalo-Fesenko, D. Hinzke, and U. Nowak, Constrained Monte Carlo method and calculation of the temperature dependence of magnetic anisotropy, Phys. Rev. B 82, 054415 (2010).

[40] C. Abert, Micromagnetics and spintronics: Models and numerical methods, Eur. Phys. J. B. 92, 120 (2019).

[41] C. Tannous and J. Gieraltowski, The Stoner-Wohlfarth model of ferromagnetism, Eur. J. Phys. 29, 475 (2008). 